\title{
Perfil nutricional de espécies lenhosas de duas florestas semidecíduas em Uberlândia, MG
}

\author{
MUNDAYATAN HARIDASAN ${ }^{1}$ e GLEIN M. ARAÚJO' ${ }^{2,3}$
}

(recebido: 12 de fevereiro de 2004; aceito: 13 de janeiro de 2005)

\begin{abstract}
Nutritional status of woody species of two semideciduous forests in Uberlândia, MG). The objective of this study was to determine the influence of soil fertility on the mineral nutrition and distribution of native species in two semideciduous forests at the Experimental Farm at Gloria (FEG) and the Ecological Station at Panga (EEP) in Uberlândia, MG. Leaf samples were collected from two to four individuals with a minimum circumference of $10 \mathrm{~cm}$ at $1,30 \mathrm{~m}$ height of all species within 50 contiguous plots of $10 \mathrm{~m} \times 10 \mathrm{~m}$ in each forest. Soil samples were analyzed from the A1 e A3 horizons of each plot of both areas. The soil of EEP showed lower acidity, higher availability of $\mathrm{Ca}$ and lower availability of $\mathrm{Al}, \mathrm{Fe}, \mathrm{Zn}$ and $\mathrm{Cu}$. The availability of $\mathrm{Mn}$ was lower in the A3 horizon and higher in the A1 horizon at EEP. The foliar concentrations of Ca e K were higher at EEP which reflected the higher levels in soil. There were no differences in the concentrations of $\mathrm{P}, \mathrm{Mg}, \mathrm{Fe}, \mathrm{Cu}$ and $\mathrm{Zn}$. The $\mathrm{N}$ concentrations were higher among species occurring exclusively at FEG. The Mn concentrations were lower at EEP, reflecting the effect of $\mathrm{pH}$ on its absorption. Thus the occurrence of some species exclusively at EEP seemed to be associated with their ability to absorb $\mathrm{K}$ and $\mathrm{Mg}$ from soils with high $\mathrm{Ca}$ availability.
\end{abstract}

Key words - aluminum accumulators, calcicoles, foliar nutrient concentrations, soil fertility, tropical forests

RESUMO - (Perfil nutricional de espécies lenhosas de duas florestas semidecíduas em Uberlândia, MG). O estudo teve como objetivo determinar a influência da fertilidade sobre nutrição mineral e distribuição das espécies nativas em duas florestas semidecíduas na Fazenda Experimental de Glória (FEG) e Estação Ecológica do Panga (EEP) em Uberlândia, MG. Foram amostradas de duas a quatro árvores, com circunferência mínima de $10 \mathrm{~cm}$, a altura de $1,30 \mathrm{~m}$ do solo, de todas as espécies encontradas em 50 parcelas contíguas de $10 \mathrm{~m} \times 10 \mathrm{~m}$, em cada floresta. Foram analisadas amostras de solo dos horizontes A1 e A3 de cada parcela nas duas florestas. $\mathrm{O}$ solo da EEP apresentou menor acidez, associada à maior disponibilidade de Ca e menor disponibilidade de Al, Fe, Zn e Cu. A disponibilidade Mn foi menor no horizonte A3 e maior no A1 na EEP. As concentrações foliares de $\mathrm{Ca}$ e $\mathrm{K}$ foram maiores nas espécies da EEP. As diferenças no teor de Ca refletiram a maior disponibilidade do nutriente no solo. Não houve diferenças nas concentrações foliares de Pe Mg. O teor médio de N foi maior na floresta FEG do que na EEP, apenas em espécies exclusivas. As concentrações de Mn foram menores na EEP, refletindo a influência do pH na absorção deste nutriente. Assim, a ocorrência de espécies presentes exclusivamente na EEP pode estar associada à capacidade de absorver $\mathrm{Ke} \mathrm{Mg}$ de solos com altos teores de $\mathrm{Ca}$.

Palavras-chave - acumuladoras de alumínio, calcícolas, concentração foliar de nutrientes, fertilidade do solo, floresta tropical

\section{Introdução}

A caracterização de uma comunidade vegetal quanto à nutrição mineral e às suas interações com características do solo pode ser feita utilizando-se a análise foliar. Essa técnica, no entanto, constitui um dos grandes desafios da Ecologia Vegetal. Sabe-se que as espécies apresentam diferenças entre si quanto ao teor de nutrientes foliares, mesmo em ambientes edáficos praticamente iguais (Haridasan 1987, 2000), mas, mesmo assim, podem refletir a disponibilidade de nutrientes e

1. Universidade de Brasília, Departamento de Ecologia, Caixa Postal 04457, 70904-970 Brasília, DF, Brasil.

2. Universidade Federal de Uberlândia, Instituto de Biologia, 38400-902 Uberlândia, MG, Brasil.

3. Autor para correspondência: glein@ufu.br as características dos solos sobre os quais se encontram (Haridasan \& Araújo 1988, Haridasan 2001). A maioria dos trabalhos sobre teor de mineral foliar, em espécies vegetais, foi realizada em florestas de latitudes médias, dentre os quais os de Guha \& Mitchell (1966), Chapin III (1980) e Ricklefs \& Mathew (1982) serviram de exemplo para estudos na região tropical.

Na América Tropical, maior número de estudos relaciona-se à ciclagem de nutrientes, sendo realizados, principalmente, na Região Amazônica (Klinge \& Rodrigues 1968, Golley et al. 1978, 1980, Golley 1986). Nesses estudos, foram analisadas amostras compostas de folhedo, não permitindo conhecer a contribuição de cada espécie na ciclagem de nutrientes. Aqueles que analisaram o teor de nutrientes em folhas adultas de espécies arbóreas foram os de Tanner (1977) na Jamaica e de Montes \& Medina (1977), Sobrado \& Medina (1980) e Medina et al. (1990) na Venezuela. 
No Brasil, a maior parte das análises da concentração de nutrientes nas folhas de comunidades vegetais nativas foi feita em cerrados, com ênfase em espécies lenhosas acumuladoras de $\mathrm{Al}$ (Haridasan 1982, 1987, Araújo \& Haridasan 1988). Os mais recentes (Haridasan 2000, Lilienfein et al. 2001) continuam desvendando o intrigante comportamento das espécies quanto à nutrição mineral nos cerrados e em comunidades vegetais florestais dessa região (Marimon et al. 2001). Os cerrados, assim como as florestas, estão sofrendo rápida transformação e é inegável a necessidade de maiores informações sobre as condições nutricionais de suas espécies, em diferentes tipos de solos.

A hipótese deste trabalho foi que as duas comunidades arbóreas, ocorrendo em solos originários de calcário e arenito, com diferentes níveis de cálcio e acidez, apresentariam perfis nutricionais distintos. O estudo teve como objetivo determinar a disponibilidade de nutrientes nos solos e as concentrações destes nas folhas em espécies nativas em duas florestas, uma na Fazenda Experimental de Glória (FEG) e outra na Estação Experimental de Panga (EEP), Uberlândia, MG, contribuindo, assim, para melhor entendimento da distribuição das espécies nativas em diferentes solos.

\section{Material e métodos}

O estudo foi realizado em duas florestas semidecíduas, uma na Estação Ecológica do Panga (EEP) e outra na Fazenda Experimental da Glória (FEG), ambas pertencentes à Universidade Federal de Uberlândia, Uberlândia, Minas Gerais. A EEP (19¹0' S e 48 $23^{\prime} \mathrm{W}$ ) possui uma área de 409,5 ha (39 ha de floresta) e localiza-se na parte Sul do Município de Uberlândia, a $35 \mathrm{~km}$ do centro da cidade (Schiavini \& Araújo 1989). A FEG (1857' S e 48¹2' W) situa-se a $12 \mathrm{~km}$ do centro de Uberlândia e possui área de $685 \mathrm{ha}$. A maior parte da fazenda destina-se a atividades agropastoris, tendo uma reserva de floresta com cerca de 30 ha. Araújo \& Haridasan (1997) encontraram 93 espécies arbóreas na EEP e 96 espécies na FEG (38 comuns às duas áreas) em um levantamento fitossociológico em 50 parcelas de $10 \mathrm{~m} \times$ $10 \mathrm{~m}$ em cada floresta. Indivíduos lenhosos com circunferência a $1,30 \mathrm{~m}$ do solo $\mathrm{t} \square 0 \mathrm{~cm}$ foram amostrados. A floresta da EEP apresentou uma densidade de 2.202 indivíduos ha ${ }^{-1}$ com área basal de $23,8 \mathrm{~m}^{2}$.ha ${ }^{-1}$ e a floresta da FEG 1.632 indivíduos com $28 \mathrm{~m}^{2} \cdot \mathrm{ha}^{-1}$.

Grande parte do Triângulo Mineiro possui clima tropical de savana, com inverno seco e verão chuvoso, sendo a temperatura média do mês mais frio superior a $18^{\circ} \mathrm{C}$. As áreas mais elevadas da região são caracterizadas pelos climas tipo Cwb e Cwa. A precipitação pluviométrica anual varia de 1.400 a $1.700 \mathrm{~mm}$ e as temperaturas médias máximas de 27 a $30^{\circ} \mathrm{C}$. $\mathrm{O}$ Município de Uberlândia possui clima tipo Cwb na parte Norte e Cwa na parte Sul, porém o mais típico dos climas do Município é o Aw (Embrapa 1982).

O Latossolo Vermelho Escuro, associado às áreas geológicas do grupo Bauru, formação Adamantina, predomina no Triângulo Mineiro e no Município de Uberlândia (Embrapa 1982), sendo de moderado a fortemente ácido. Em áreas mais restritas, são encontrados solos eutróficos, com saturação de bases entre $50 \%$ e $65 \%$, e desenvolvidos a partir de materiais originados do arenito do grupo Bauru, formação Marília, com cimento calcífero (Embrapa 1982).

Para a avaliação da fertilidade dos solos, foram retiradas amostras compostas de cada uma das 50 parcelas, estabelecidas para levantamento fitossociológico de cada floresta (Araújo \& Haridasan 1997). As coletas foram realizadas nas profundidades de 0 a $8 \mathrm{~cm}$ e 8 a $65 \mathrm{~cm}$ na EEP, e de 0 a $7 \mathrm{~cm}$ e de 7 a $24 \mathrm{~cm}$ na FEG, correspondendo aos horizontes A1 e A3, nos dois solos. As amostras foram secas ao ar e passadas por uma peneira com malha de $2 \mathrm{~mm}$. A textura do solo foi determinada pelo método de Bouyoucos (Kiehl 1979). O pH foi medido em água e KCl, 1M. Os teores

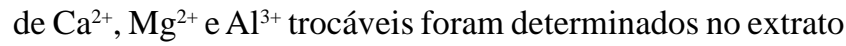
de $\mathrm{KCl}, 1 \mathrm{M}$ e os outros nutrientes $\left(\mathrm{P}, \mathrm{K}^{1+}, \mathrm{Fe}^{3+}, \mathrm{Mn}^{2+}, \mathrm{Zn}^{2+} \mathrm{e}\right.$ $\mathrm{Cu}^{2+}$ ) no extrato de Mehlich $\left(\mathrm{H}_{2} \mathrm{SO}_{4}, 0,0125 \mathrm{M}+\mathrm{HCl} 0,05 \mathrm{M}\right)$ (Allen 1974). O Al foi obtido por titulação com NaOH, 0,025M. $\mathrm{O}$ teor de $\mathrm{P}$ foi determinado por colorimetria, utilizando-se o molibdato de amônia, a $660 \mathrm{~nm}$. Os teores dos demais elementos foram medidos em espectrofotometria de absorção atômica ou emissão de chama.

Todas as 93 espécies da EEP e 96 da FEG, encontradas no levantamento de Araújo \& Haridasan (1997), foram amostradas para análise do teor de nutrientes nas folhas. Foram coletadas folhas adultas, sem lesões ou sinais de ataques de insetos, de dois a quatro indivíduos de cada espécie na segunda quinzena do mês de março de 1990, no final da estação chuvosa. Logo após a coleta, as folhas foram lavadas com água destilada e secas em estufa a $70{ }^{\circ} \mathrm{C}$. As amostras secas foram moídas em moinho tipo Wiley e acondicionadas em sacos plásticos.

$\mathrm{O}$ teor de $\mathrm{N}$ nas folhas foi determinado utilizando-se $\mathrm{o}$ método de Kjeldahl (Allen 1974). Para se obter o teor de P, K, $\mathrm{Ca}, \mathrm{Mg}, \mathrm{Mn}, \mathrm{Zn}, \mathrm{Cu}$ e $\mathrm{Al}$ as amostras de folhas foram digeridas, utilizando-se mistura triácida (ácidos nítrico, sulfúrico e perclórico), na proporção de 10:1:2 (Allen 1974). O teor de $\mathrm{P}$ no extrato de folhas foi determinado por colorimetria, a $410 \mathrm{~nm}$, utilizando-se vanadomolibdato de amônia. As concentrações dos demais nutrientes foram obtidas por espectrofotometria de absorção atômica ou emissão de chama (Allen 1974). As análises de folhas e solos foram realizadas no Laboratório de Solos, do Departamento de Ecologia da Universidade de Brasília.

Foi utilizada análise de variância, para testar diferenças entre solos e comunidades vegetais, e teste de Duncan a 5\% de probabilidade, para comparação de médias. 


\section{Resultados}

Fertilidade dos solos - A principal diferença entre os solos das duas florestas reside no maior teor de $\mathrm{Ca}$ disponível na EEP, nos dois horizontes, associado à menor acidez (figura 1). O solo da EEP, com $76 \pm 8 \%$ de saturação de bases no horizonte A1 e $49 \pm 10 \%$ no A3, é um solo eutrófico, enquanto o da FEG, com saturação de bases de $29 \pm 11 \%$ no horizonte A1 e 7,4 $\pm 3,0 \%$ no A3, é distrófico. Acompanhando estas propriedades, a saturação de $\mathrm{Al}$ foi menor no solo da $\operatorname{EEP}(0,78 \pm 0,49 \%$ no A 1 e $7,48 \pm 4,91 \%$ no A3), em comparação com o solo da FEG $(26,3 \pm 14,2 \%$ no A1 e $63,9 \pm 11,8 \%$ no A3). A disponibilidade de $\mathrm{Mg}$ e $\mathrm{K}$ foi baixa nos dois solos e não houve diferenças na disponibilidade de P. Apenas com o Mn, houve uma inversão nas diferenças entre os horizontes A1 e A3. O horizonte A1 no solo da EEP apresentou maior teor de Mn do que A1 da FEG, enquanto no horizonte A3, o solo da EEP apresentou menor teor do que a FEG (figura 1). Teor de minerais nas folhas - Os teores médios de nutrientes foliares, nas 38 espécies comuns, encontramse na figura 2. Esses dados permitem avaliar as diferenças nas concentrações foliares entre os locais, eliminando-se prováveis variações interespecíficas. $\mathrm{Na}$ figura 3 , a comparação está restrita às espécies que ocorrem exclusivamente em cada uma das áreas (55 na EEP 58 na FEG). Essa comparação permite avaliar o perfil nutricional das duas comunidades, visando discutir aspectos como: adaptação e distribuição de espécies nativas em diferentes solos.

As espécies comuns e as exclusivas, em geral, responderam de maneira semelhante à disponibilidade de nutrientes no solo e à acidez. Não houve diferenças
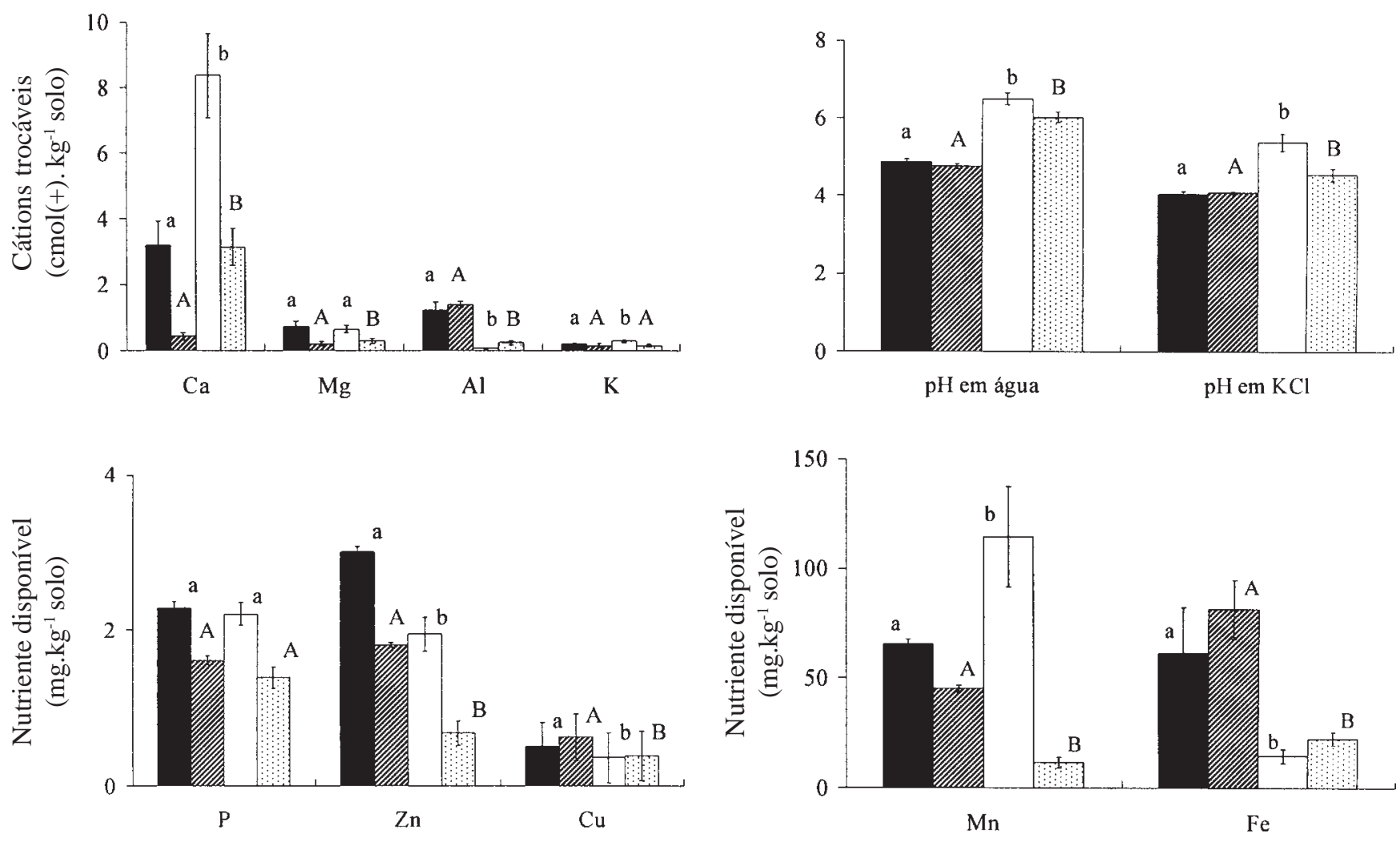

Figura 1. Propriedades dos solos (média e desvio padrão) nos horizontes A1 e A3, da floresta semidecídua da Fazenda Experimental de Glória e da Estação Ecológica do Panga, Uberlândia, MG. Médias para o mesmo elemento para as duas florestas (minúsculas para o horizonte A1 e maiúsculas para o horizonte A3) não diferem entre si pelo teste de Duncan, a 5\% de probabilidade. = A1 - Fazenda Experimental de Glória; $\square=$ A3 - Fazenda Experimental de Glória; $\square=$ A1 - Estação Ecológica do Panga; $;$ = A3 - Estação Ecológica do Panga.

Figure 1. Properties of A1 and A3 horizons of the soils (mean and standard deviation) of semideciduous forests of the Experimental Farm at Glória and the Ecological Station at Panga in Uberlândia, MG. Means for the same nutrient for the two forests followed by the same letter (lowercase for the A1 horizon and uppercase for the A3 horizon) do not differ between themselves as judged by the Duncan's test at 5\% probability level. $\mathbf{a}=\mathrm{A} 1$ - Experimental Station of Glória; $\square=\mathrm{A} 3$ Experimental Station at Glória; $\square=$ A1 - Ecological Station at Panga; $\mathrm{B}$ = A3 - Ecological Station at Panga. 

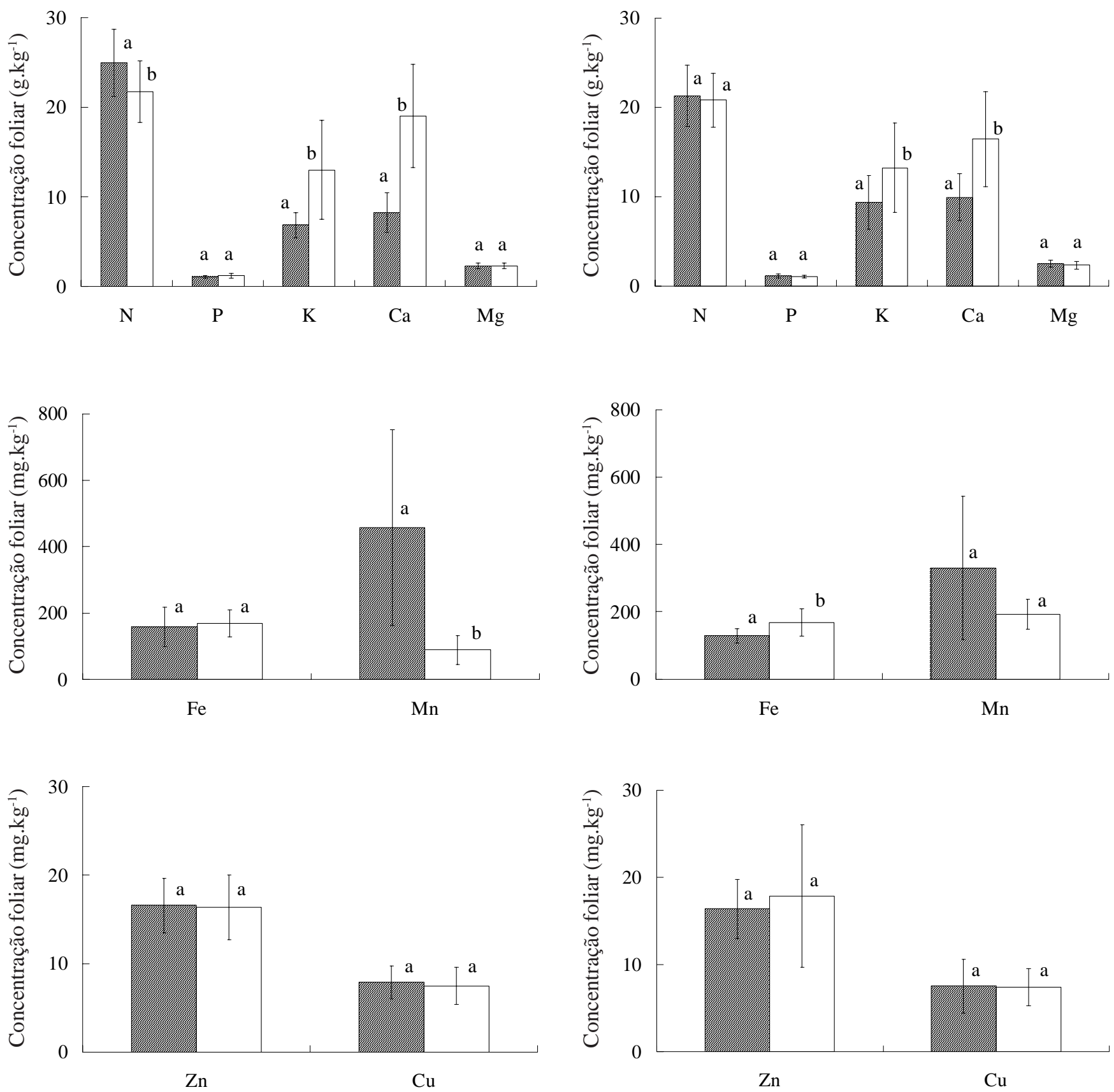

Figura 2. Concentrações foliares de nutrientes (média e desvio padrão) em espécies arbóreas comuns da floresta semidecídua da Fazenda Experimental de Glória (絗) e da Estação Ecológica do Panga ( $\square$ ), Uberlândia, MG. Médias para o mesmo nutriente com mesmas letras não diferem entre si pelo teste de Duncan, a $5 \%$ de probabilidade.

Figure 2. Foliar nutrient concentrations (mean and standard deviation) in the tree species, which are common to both semideciduous forests of the Experimental Farm at Glória (国) and the Ecological Station at Panga ( $\square$ ) in Uberlândia, MG. Means for the same nutrient followed by the same case letter do not differ between themselves as judged by the Duncan's test at $5 \%$ probability level.

Figura 3. Concentrações foliares de nutrientes (média e desvio padrão) em espécies arbóreas exclusivas da floresta semidecídua da Fazenda Experimental de Glória (路) e da Estação Ecológica do Panga ( $\square$ ), Uberlândia, MG. Médias para o mesmo nutriente com mesmas letras não diferem entre si pelo teste de Duncan, a 5\% de probabilidade.

Figure 3. Foliar nutrient concentrations (mean and standard deviation) in the tree species which are exclusive to each semideciduous forest of the Experimental Farm at Glória (罌) and the Ecological Station at Panga ( $\square$ ) in Uberlândia, MG. Means for the same nutrient followed by the same case letter do not differ between themselves as judged by the Duncan's test at $5 \%$ probability level. 
significativas nas concentrações foliares de $\mathrm{Pe} \mathrm{Mg}$ entre as duas comunidades, refletindo a similaridade entre os dois solos em relação a esses nutrientes. As diferenças maiores e mais consistentes foram nos teores de $\mathrm{Ca} \mathrm{e}$ $\mathrm{K}$, sendo maiores as concentrações nas espécies das comunidades da EEP (figuras 2, 3). Na FEG, 95\% delas tiveram concentrações de Ca entre 6,3 e 14,8 g. $\mathrm{kg}^{-1}$, enquanto que na EEP em $30 \%$ das espécies foram detectados teores maiores. As mais ricas em Ca na FEG, Celtis iguanae (Jacq.) Sargent, Maclura tinctoria D.Don ex Steud., Miconia sp. e Myrsine guianensis (Aubl.) Kuntze, tiveram teores de 23,8 a 32,7 g.kg-1. Concentrações acima de 44 g. kg ${ }^{-1}$ foram determinadas em Celtis iguanae (Jacq.) Sargent, Dendropanax cuneatum (DC.) Decne \& Planch., Eugenia stricta Pranch. ex Bronq. \& Griseb. e Rhamnidium elaeocarpum Reisseck, todas da floresta da EEP.

As espécies comuns e exclusivas da EEP apresentaram menores teores de Mn do que da FEG (figuras 2, 3), apesar do solo da floresta da EEP ter maior disponibilidade desse micronutriente no horizonte A1 (figura 1). Variações interespecíficas foram acentuadas entre as espécies da FEG, onde foram encontradas 33 espécies com mais de $300 \mathrm{mg} \cdot \mathrm{kg}^{-1}$ de Mn (tabela 1), com os maiores valores acima de $2.000 \mathrm{mg} \cdot \mathrm{kg}^{-1}$. As variações nas concentrações de outros micronutrientes $(\mathrm{Fe}, \mathrm{Zn} \mathrm{e} \mathrm{Cu}$ ) foram as menores entre os elementos analisados.

Quanto ao teor de $\mathrm{Al}$ nas folhas, 83,7\% das espécies das duas florestas tiveram valores de 33 a $132 \mathrm{mg} . \mathrm{kg}^{-1}$. Foram encontradas 9 espécies acumuladoras de $\mathrm{Al}$ com teores superiores a $1.000 \mathrm{mg} \cdot \mathrm{kg}^{-1}$, sendo quatro exclusivas da FEG e duas da EEP e três comuns às duas áreas (tabela 2). Os maiores teores foram obtidos em Coussarea contracta (Walp.) Müll.Arg. (4.185 mg. $\mathrm{kg}^{-1}$ ) na FEG e $C$. hydrangeaefolia Benth. \& Hook. (3.650 mg.kg $\left.{ }^{-1}\right)$ na EEP.

\section{Discussão}

O maior teor de Ca no solo eutrófico da EEP é devido à sua origem de arenitos do Grupo Bauru, formação Marília, com cimentação carbonática (Nishiyama 1989). De modo geral, os solos originados de rocha basáltica ou calcária apresentam também maiores teores de Mn (Krauskopf 1972). O solo da FEG é originário de sedimentos cenozóicos. Solos desta origem no Município de Uberlândia são geralmente distróficos e suportam florestas (Embrapa 1982, Araújo et al. 1997, Guimarães et al. 2001). Assim, os dois locais escolhidos para comparação de perfil nutricional de florestas representam condições edáficas distintas. A presença de florestas em solos eutróficos e distróficos e suas divergências em relação à composição florística e fitossociologia na região dos cerrados são bem documentadas na literatura (Ratter et al. 1977, 1978 a, b). As diferenças nestes aspectos, entre as duas florestas, foram discutidas em Araújo \& Haridasan (1997).

A comparação da fertilidade dos solos é talvez mais simples neste caso do que a comparação do perfil nutricional das duas florestas. Os conceitos de disponibilidade de nutrientes no solo e os efeitos de variação do $\mathrm{pH}$ sobre a toxicidade de elementos como $\mathrm{Al}, \mathrm{Fe}$ e Mn são consensos em relação à agricultura e plantas cultivadas (Marschner 1995). Em geral, as plantas respondem à maior disponibilidade de macronutrientes, sendo as leguminosas uma exceção no caso de nitrogênio. É bem conhecido também que a proporção de cátions $\mathrm{K}, \mathrm{Ca}$ e $\mathrm{Mg}$ no solo influencia a absorção destes pelas plantas. A toxicidade de Al, Fe e Mn às plantas que ocorrem em comunidades nativas pode não ser verificada em solos ácidos e distróficos, uma vez que elas possivelmente sejam adaptadas a esses ambientes, sem prejuízo ao seu crescimento e produção. Por outro lado, isso pode ser um fator que causa a exclusão de algumas espécies de solo ácido.

A comparação dos perfis nutricionais das duas florestas, ao contrário dos solos, necessita uma análise mais cuidadosa, devido às diferenças na composição florística. Esta questão foi abordada por Haridasan \& Araújo (1988), quando foram comparados dois cerradões, em solos distrófico e mesotrófico, em relação às concentrações foliares de nutrientes. Ao invés de se comparar os valores médios de todas as espécies, uma alternativa é separar as espécies que ocorrem nas duas comunidades em dois grupos: aquelas que aparecem nos dois locais e aquelas presentes exclusivamente em um dos locais. A justificativa para comparar as espécies comuns é que as diferenças observadas nas concentrações foliares podem ser atribuídas diretamente às diferenças na fertilidade e outros fatores edáficos, eliminando as diferenças intraespecíficas. Por outro lado, uma comparação entre as espécies exclusivas pode apontar diferenças entre as comunidades, quanto à adaptação e à distribuição das espécies nativas aos ambientes específicos. Além disso, pode-se explicar melhor as eventuais diferenças no funcionamento dos dois ecossistemas em relação à nutrição mineral das plantas e à ciclagem de nutrientes.

A diferença nas concentrações foliares de $\mathrm{Ca}$, tanto em espécies comuns como em exclusivas deste estudo, 
Tabela 1. Concentração foliar de $\mathrm{Mn}\left(\mathrm{mg} \cdot \mathrm{kg}^{-1}\right)$ nas espécies acumuladoras em florestas semidecíduas da Fazenda Experimental de Glória (FEG) e da Estação Ecológica do Panga (EEP) em Uberlândia, MG.

Table 1. Foliar concentration of $\mathrm{Mn}\left(\mathrm{mg} \cdot \mathrm{kg}^{-1}\right)$ in accumulator species of semideciduous forests of the Experimental Farm at Glória (FEG) and the Ecological Station at Panga (FEG) in Uberlândia, MG.

\begin{tabular}{|c|c|c|}
\hline Espécies & FEG & $\mathrm{EEP}$ \\
\hline \multicolumn{3}{|l|}{ Espécies comuns } \\
\hline \multicolumn{2}{|c|}{ Agonandra brasiliensis Benth. \& Hook. 651} & 130 \\
\hline Casearia sylvestris $\mathrm{Sw}$. & 925 & 384 \\
\hline Copaifera langsdorffii Desf. & 709 & 452 \\
\hline Cupania vernalis Camb. & 1.501 & 598 \\
\hline Hymenaea courbaril L. & 423 & 356 \\
\hline Luehea paniculata Mart. \& Zucc. & 468 & 396 \\
\hline Ouratea castaneaefolia (DC.) Engl. & 1.734 & 698 \\
\hline Protium heptaphyllum (Aubl.) March. & 1. 384 & 122 \\
\hline Rheedia gardneriana Planch. \& Trianء & na 897 & 621 \\
\hline Roupala brasiliensis Klotz. & 503 & 431 \\
\hline Virola sebifera Aubl. & 1.304 & 790 \\
\hline Média \pm Desvio padrão & $863 \pm 463$ & $452 \pm 213$ \\
\hline \multicolumn{3}{|l|}{ Espécies exclusivas } \\
\hline Actinostemon communis $\mathrm{Pax}$ & 835 & \\
\hline Casearia decandra Jacq. & 883 & \\
\hline Casearia grandiflora Camb. & 1.420 & \\
\hline $\begin{array}{l}\text { Coussarea contracta (Walp.) } \\
\text { Müll.Arg. }\end{array}$ & 2.477 & \\
\hline Croton floribundus Spreng. & 1.349 & \\
\hline Cryptocaria aschersoniana $\mathrm{Mez}$ & 830 & \\
\hline $\begin{array}{l}\text { Schefflera morototoni (Aubl.) } \\
\text { Maguire, Steyerm. \& Frodin }\end{array}$ & 391 & \\
\hline Duguetia lanceolata A.St.-Hil. & 490 & \\
\hline Heisteria ovata Benth. & 1.496 & \\
\hline Machaerium nyctitans (Vell.) Benth. & 1.098 & \\
\hline Machaerium sp. & 486 & \\
\hline Maytenus sp. & 921 & \\
\hline Myrcia sp. & 1.511 & \\
\hline Piptocarpha macropoda (DC.) Baker & 923 & \\
\hline Platycyamus regnellii Benth. & 2.754 & \\
\hline Psidium rufum Mart. ex DC. & 351 & \\
\hline Siparuna guianensis Aubl. & 508 & \\
\hline Siphoneugenia densiflora Berg & 831 & \\
\hline Sloanea monosperma Vell. & 859 & \\
\hline Sorocea bonplandii Lanj. \& Boer & 928 & \\
\hline Tabebuia serratifolia (Vahl.) Nicholson & n 458 & \\
\hline Vitex polygama Cham. & 881 & \\
\hline Casearia gossypiosperma Briq. & & 531 \\
\hline $\begin{array}{l}\text { Endlicheria paniculata (Spreng.) } \\
\text { Macbr. }\end{array}$ & & 310 \\
\hline
\end{tabular}

Média \pm Desvio padrão

$1.031 \pm 619420 \pm 156$
Tabela 2. Concentração foliar de $\mathrm{Al}\left(\mathrm{mg} \cdot \mathrm{kg}^{-1}\right)$ nas espécies acumuladoras em florestas semidecíduas da Fazenda Experimental de Glória (FEG) e da Estação Ecológica do Panga (EEP) em Uberlândia, MG.

Table 2. Foliar concentration of $\mathrm{Al}\left(\mathrm{mg} \cdot \mathrm{kg}^{-1}\right)$ in accumulator species of semideciduous forests of the Experimental Farm at Glória (FEG) and the Ecological Station at Panga (EEP) in Uberlândia, MG.

\begin{tabular}{|c|c|c|}
\hline Espécies & FEG & EEP \\
\hline \multicolumn{3}{|l|}{ Espécies comuns } \\
\hline Faramea cyanea Müll.Arg. & 1.902 & 2.072 \\
\hline $\begin{array}{l}\text { Rudgea viburnoides (Cham.) } \\
\text { Benth. }\end{array}$ & 1.243 & 2.002 \\
\hline Vochysia tucanorum Mart. & 3.138 & 3.459 \\
\hline Média \pm Desvio padrão & $1902 \pm 926$ & $2072 \pm 822$ \\
\hline \multicolumn{3}{|l|}{ Espécies exclusivas } \\
\hline Callisthene major Mart. & 1.200 & \\
\hline $\begin{array}{l}\text { Coussarea contracta (Walp.) } \\
\text { Müll.Arg. }\end{array}$ & 4.158 & \\
\hline Miconia sp. & 2.783 & \\
\hline Qualea jundiahy Warm. & 1.813 & \\
\hline $\begin{array}{l}\text { Coussarea hydrangeaefolia } \\
\text { Benth. \& Hook. }\end{array}$ & & 3.650 \\
\hline $\begin{array}{l}\text { Symplocos platyphylla (Pohl) } \\
\text { Benth. }\end{array}$ & & 1.268 \\
\hline Média \pm Desvio padrão & $2.298 \pm 1.290$ & $2.459 \pm 1.684$ \\
\hline
\end{tabular}

reflete diretamente as diferenças na disponibilidade deste elemento no solo. As espécies que ocorrem exclusivamente na EEP, com maior freqüência, podem ser consideradas calcícolas e as que ocorrem só na FEG, calcífugas. Um motivo para essa separação pode ser a capacidade das espécies calcícolas da EEP de absorver $\mathrm{K}$ e $\mathrm{Mg}$ do solo com alto teor de $\mathrm{Ca}$, uma vez que os dois solos apresentam a mesma disponibilidade de $\mathrm{Ke}$ $\mathrm{Mg}$. Os efeitos antagônicos de altos teores de $\mathrm{Ca}$ no solo na absorção de $\mathrm{K}$ e $\mathrm{Mg}$ são bem documentados na literatura (Marschner 1995).

Hou \& Merkle (1950) estudaram 31 espécies de solos fortemente ácidos e de solos ricos em $\mathrm{Ca}$ e sugeriram que as calcícolas seriam aquelas que possuíam concentrações entre 15,7 a 22,7 g.kg-1 e as calcífugas entre 4,8 a 10,8 g.kg-1. Pelo critério destes autores, 42 espécies da EEP podem ser consideradas calcícolas. Apenas cinco espécies da floresta da FEG, incluindo duas comuns, satisfazem este critério. Celtis iguanae (Jacq.) Sargent e Terminalia brasiliensis Raddi apresentaram altas concentrações de $\mathrm{Ca}$, nos dois solos. Estas 
observações estão de acordo com o relato de Steele (1955), que calcícolas específicas ocorrem em solos ricos em $\mathrm{Ca}$ com $\mathrm{pH}$ entre 6,5 e 7,0, mas podem ser encontradas também em solos com menor $\mathrm{pH}$. Vale ressaltar a observação de Crawley (1997), que as calcícolas podem não ser plantas com altas necessidades de $\mathrm{Ca}$, mas aquelas que são sensíveis aos fatores como a alta acidez, baixa disponibilidade de outros nutrientes e toxicidade do $\mathrm{Al}$, em solos pobres em Ca. No entanto, este parece não ser o caso das espécies da FEG, já que 78 das 96 espécies da FEG tiveram teores menores de 10,8 g. $\mathrm{kg}^{-1}$ de Ca nas folhas, podendo ser consideradas calcífugas de fato. Embora exista a possibilidade de que as baixas concentrações foliares de Ca simplesmente reflitam os baixos teores de Ca no solo, não há registros de ocorrência de indivíduos destas espécies em solos com altos teores de Ca. Ratter et al. (1977, 1978a, b) identificaram várias espécies indicadoras de solos ricos em Ca no Brasil central. Anadenanthera macrocarpa (Benth.) Brenan, Aspidosperma subincanum Mart., Celtis iguanae (Jacq.) Sargent, Dilodendron bipinnatum Radlk., Guazuma ulmifolia Lam. e Lithrea molleoides (Vell.) Engl. foram encontradas na floresta da EEP e são citadas como calcícolas por Ratter et al. (1978 b).

As menores concentrações foliares de $\mathrm{Mn}$, nas espécies comuns às duas florestas e exclusivas à EEP, refletiram a influência da menor acidez do solo, mesmo considerando-se a maior disponibilidade deste elemento no horizonte superficial (A1) na EEP. É interessante notar que os valores médios de Mn nas folhas de espécies da FEG estiveram acima do limite de $300 \mathrm{mg} \cdot \mathrm{kg}^{-1}$, estabelecido para espécies acumuladoras deste elemento por Gauch (1972), apesar de sua disponibilidade no solo ser considerada deficiente (valores inferiores a $50 \mathrm{mg} . \mathrm{kg}^{-1}$ ) conforme Marschner (1995). Assim, 33 das 96 espécies (22 exclusivas e 11 comuns) da FEG seriam acumuladoras de Mn (tabela 1). Apenas 11 das 93 espécies da EEP seriam classificadas como acumuladoras, sendo 9 dessas espécies comuns a FEG. Assim, são claras as diferentes estratégias entre as plantas nativas para superar a toxicidade de Mn em solos ácidos. Enquanto um terço das espécies da FEG (33 das 96) acumula Mn acima de $300 \mathrm{mg} \cdot \mathrm{kg}^{-1}$ até $2.750 \mathrm{mg} \cdot \mathrm{kg}^{-1}$, os outros dois terços evitam a absorção desse elemento em níveis elevados. Por outro lado, apenas duas das 55 espécies exclusivas da EEP foram acumuladoras. As nove espécies que apresentaram elevados teores de Mn, nos dois solos, seriam acumuladoras obrigatórias. Haridasan \& Araújo (1988) também verificaram que as espécies de cerradão em solo mesotrófico limitaram a absorção de Mn em solo com altos teores de $\mathrm{Ca}$, enquanto apresentaram altos teores em solo distrófico mais ácido.

A ocorrência de espécies acumuladoras de $\mathrm{Al}$ em cerrado e cerradão foi discutida por Haridasan (1982, 1987) e Haridasan \& Araújo (1988). A origem evolutiva desta característica nas plantas superiores e os mecanismos de absorção, translocação e deposição em vários componentes da biomassa estão sendo discutidos na literatura recente (Hodson \& Sangster 1999, Ma et al. 2001, Watanabe \& Osaki 2002, Jansen et al. 2002, 2003). Das nove espécies encontradas com concentrações de $\mathrm{Al}$ acima de $1.000 \mathrm{mg} \cdot \mathrm{kg}^{-1}$, neste estudo, cinco ocorreram na EEP em solo rico em Ca e baixa saturação de $\mathrm{Al}$, sendo três comuns a FEG. Isso reforça a hipótese anterior de que a distribuição de acumuladoras de Al não está restrita aos solos fortemente ácidos e distróficos (Haridasan \& Araújo 1988). Entretanto, as densidades delas nas duas comunidades foram menores do que as de outras espécies acumuladoras encontradas em comunidades vegetais de cerrado (sentido restrito) e cerradão, em solos distróficos no Planalto Central (Haridasan 1987).

\section{Referências bibliográficas}

ALLEN, S.E. 1974. Chemical Analysis of Ecological Materials. Blackwell Scientific Publications, Oxford.

ARAÚJO, G.M. \& HARIDASAN, M. 1988. A comparison of the nutritional status of two forest communities on mesotrophic and dystrophic soils in central Brazil. Communications in Soil Science and Plant Analysis 19:1075-1089.

ARAÚJO, G.M. \& HARIDASAN, M. 1997. Estrutura fitossociológica de duas matas mesófilas semidecíduas, em Uberlândia, Triângulo Mineiro. Naturalia 22:115-129.

ARAÚJO, G.M., GUIMARÃES, A.J.M. \& NAKAJIMA, J.N. 1997. Fitossociologia de um remanescente de mata mesófila semidecídua urbana, Bosque John Kennedy, Araguari, MG, Brasil. Revista Brasileira de Botânica 20:67-77.

CHAPIN III, F.S. 1980. The mineral nutrition of wild plants. Annual Review of Ecology and Systematics 11:233-260.

CRAWLEY, M.J. 1997. Life history and environment. In Plant Ecology (M.J. Crawley, ed.). Blackwell Science, London, p.73-131.

EMBRAPA. 1982. Levantamento de reconhecimento de média intensidade dos solos e avaliação da aptidão agrícola das terras do Triângulo Mineiro. Boletim Técnico $\mathrm{n}^{\circ} 1$, Serviço Nacional de Levantamento e Conservação de solos, Rio de Janeiro. 
GAUCH, H.G. 1972. Inorganic plant nutrition. Dowden, Hutchinson \& Ross, Stroudsberg.

GOLLEY, F.B. 1986. Chemical plant-soil relationships in tropical forests. Journal of Tropical Ecology 2:219-229.

GOLLEY, F.B., RICHARDSON, T. \& CLEMENTS, R.G. 1978. Elemental concentrations in tropical forests and soils of northwestern Colombia. Biotropica 10:144-151.

GOLLEY, F.B., YANTKO, J., RICHARDSON, T. \& KLINGE, H. 1980. Biogeochemistry of tropical forests: 1 . The frequency distribution and mean concentration of selected elements in a forest near Manaus, Brazilian. Tropical Ecology 21:59-70.

GUHA, M.M. \& MITCHELL, R.L. 1966. The trace and major element composition of the leaves of some deciduous trees II. Seasonal changes. Plant and Soil 24:90-112.

GUIMARÃES, A.J.M., CORRÊA, G.F. \& ARAÚJO, G.M. 2001. Características da vegetação e do solo em duas comunidades vegetais contíguas no Triângulo Mineiro. Boletim do Herbário Ezechias Paulo Heringer 7:113-127.

HARIDASAN, M. 1982. Aluminium accumulation by some cerrado native species of central Brazil. Plant and Soil 65:265-273.

HARIDASAN, M. 1987. Distribution and mineral nutrition of aluminium-accumulating species in different plant communities of the cerrado region of Central Brazil. In La capacidad bioproductiva de sabanas (J.J. San José \& R. Montes, eds.). Instituto Venezolano de Investigaciones Cientificas, Caracas, p.309-348.

HARIDASAN, M. 2000. Nutrição mineral de plantas nativas do cerrado. Revista Brasileira de Fisiologia Vegetal 12:54-64.

HARIDASAN, M. 2001. Nutrient cycling as a function of landscape and biotic characteristics in the cerrado of central Brazil. In Biogeochemistry of the Amazon basin and its role in a changing world (M.E. McClain, R.L. Victoria \& J.E. Richey, eds.). Oxford University Press, New York. p.68-83.

HARIDASAN, M. \& ARAÚJO, G.M. 1988. Aluminiumaccumulating species in two forest communities in the cerrado region of central Brazil. Forest Ecology and Management 24:15-26.

HODSON, M.J. \& SANGSTER, A.G. 1999. Aluminium/Silicon interactions in conifers. Journal of Inorganic Biochemistry 76:89-98.

HOU, H.Y. \& MERKLE, F.G. 1950. Chemical composition of certain calcifugous and calcicole plants. Soil Science 69:471-486.

JANSEN, S., BROADLEY, M., ROBBRECHT, E. \& SMETS, E. 2002. Aluminium hyperaccumulation in angiosperms: a review of its phylogenetic significance. Botanical Review 68:235-269.

JANSEN, S., SMETS, E. \& HARIDASAN, M. 2003. Aluminum accumulation in flowering plants. In McGraw-Hill Yearbook of Science and Technology (D. Blumel \& A. Rappaport, eds.). McGraw-Hill, New York, p.11-13.
KIEHL, E.J. 1979. Manual de Edafologia. Editora Agronômica Ceres, São Paulo.

KLINGE, H. \& RODRIGUES, W.A. 1968. Litter production in an area of Amazonian terra firme forest. Part I. Litter fall, organic carbon and total nitrogen contents of litter. Amazoniana 1:287-302.

KRAUSKOPF, K.B. 1972. Geochemistry of micronutrients. In Micronutrients in agriculture (J.J. Mortvedt, P.M. Giordano \& W.L Lindsay, eds.). Soil Science Society of America, Madison. p.7-40.

LILIENFEIN, J., WILCKE, W., ZIMMERMANN, R., GERSTBERGER, P., ARAÚJO, G.M. \& ZECH, W. 2001. Nutrient storage in soil and biomass of native Brazilian Cerrado. Journal Plant Nutrition and Soil Science 164:487-495.

MA, J.F., RYAN, P.R. \& DELHAIZE, E. 2001. Aluminum tolerance in plants and the complexing role of organic acids. Trends in Plant Science 6:273-278.

MARIMON, B.S., FELFILI, J.M. \& HARIDASAN, M. 2001. Studies in monodominant forests in eastern Mato Grosso, Brazil: II. A forest in the areões Xavante Indian Reserve. Edinburgh Journal of Botany 58:1-15.

MARSCHNER, H. 1995. Mineral nutrition of higher plants. Academic Press, New York.

MEDINA, E., GARCIA, V. \& CUEVAS, E. 1990. Sclerophylly and oligotrophic environments: relationships between leaf structure, mineral nutrient content, and drought resistance in tropical rain forests of the upper Rio Negro region. Biotropica 22:51-64.

MONTES, R. \& MEDINA, E. 1977. Seasonal changes in nutrient of leaves of savanna trees with different ecological behavior. Geografia y Ecologia Tropical 4:295-307.

NISHIYAMA, L. 1989. Geologia do município de Uberlândia e áreas adjacentes. Sociedade e Natureza 1:9-16.

RATTER, J.A., ASKEW, G.D., MONTGOMERY, R.F. \& GIFFORD, D.R. 1977. Observações adicionais sobre o cerradão de solos mesotróficos no Brasil Central. In IV Simpósio sobre o Cerrado. Edusp, São Paulo, p.306-316.

RATTER, J.A., ASKEW, G.P., MONTGOMERY, R.F. \& GIFFORD, D.R. 1978a. Observation on the vegetation of northeastern Mato Grosso II. Forest and soil of the Rio Suia-Missu area. Proceedings of the Royal Society, London 203:191-208.

RATTER, J.A., FURLEY, P.A., MONTGOMERY, R.F. \& GIFFORD, D.R. 1978b. Observations on forests of some mesotrophic soils in Central Brazil. Revista Brasileira de Botânica 1:47-58.

RICKLEFS, R.E. \& MATHEW, K.K. 1982. Chemical characteristics of the foliage of some deciduous trees in southeastern Ontario. Canadian Journal of Botany 60:2037-2045.

SCHIAVINI, I. \& ARAÚJO, G.M. 1989. Considerações sobre a vegetação da Reserva Ecológica da EEP (Uberlândia). Sociedade e Natureza 1:61-66. 
SOBRADO, M.A. \& MEDINA, E. 1980. General morphology, anatomical structure, and nutrient content of sclerophyllous leaves of the "Bana" vegetation of Amazonas. Oecologia 45:341-345.

STEELE, B. 1955. Soils $\mathrm{pH}$ and base status as factors on the distribution of calcicoles. Journal of Ecology 43:120-132.
TANNER, E.V.J. 1977. Four montane rain forests of Jamaica: a quantitative characterization of floristics, the soils and the foliar mineral levels and a discussion of the interrelations. Journal of Ecology 65:883-919.

WATANABE, T. \& OSAKI, M. 2002. Mechanisms of adaptation to high aluminum condition in native plant species growing in acid soils: a review. Communications in Soil Science and Plant Analysis 33:1247-60. 